\title{
Forward Secure ID-based Group Key Agreement Protocol with Anonymity
}

\author{
Hyewon Park, Zeen Kim, and Kwangjo Kim \\ Department of Information and Communications Engineering, \\ Korea Advanced Institute of Science and Technology (KAIST), \\ 119 Munjiro, Yuseong-gu, Daejeon, 305-732 Korea. \\ \{inde1776, zeenkim, kimkj\}@kaist.ac.kr
}

\begin{abstract}
ID-based group key agreement (GKA) has been increasingly researched with the advantage of simple public key management. However, identities of group members can be exposed in the ID-based GKA protocol, so eavesdroppers can easily learn who belongs to the specific group. Recently, Wan et al. [7] proposed a solution for this problem, an anonymous ID-based GKA protocol, which can keep group members' anonymity to outside eavesdroppers; nevertheless, the protocol has some security flaws.

This paper shows that Wan et al.'s GKA is insecure against colluding attack and their joining/leaving protocols do not guarantee forward and backward secrecy. We also propose a new forward secure ID-based GKA with anonymity from enhancing Wan et al.'s joining/leaving protocols. Our scheme provides forward and backward secrecy and is essentially just efficient as Wan et al.'s scheme.
\end{abstract}

\section{Introduction}

In modern society, many group-oriented applications exist, such as Internet conferencing, chatting, or collaborative workspace. These applications usually require privacy for communication messages; that is, all the messages exchanged during communication should be protected from eavesdroppers. Therefore, the group members need a common secret key to encrypt their communication messages. Group key agreement (GKA) is the process that the legitimated group members share a group key; no users can predetermine the final group key.

Recently, ID-based GKA has been increasingly proposed with the advantage of an ID-based cryptosystem [1]. In IDbased cryptosystem, user's identity information, e.g. email adderess or PIN number, is used as public keys, and a key generation center (KGC) generates the corresponding private keys; therefore, no certificate is needed to bind user names with their public keys. Though ID-based GKA protocols([3], [5], [8], [6]) have the advantage in simple key management, it has one serious problem that cannot provide privacy of group members. In other words, the identities of group members are exposed to eavesdroppers during the protocol execution.
In 2008, Wan et al.[7] proposed an anonymous IDbased GKA protocol. Their protocol keeps the advantage of the ID-based cryptosystem and provides anonymity of the identities from outsiders. The authors also proposed joining and leaving protocols for dynamic operation of a single user.

In this paper, we show that Wan et al.'s GKA protocol is insecure in the presence of malicious participants. Two malicious neighbors of a specific user who can collude with the group initiator can impersonate the user during the group execution. In addition, their joining protocol cannot provide group backward secrecy, so joining members can get the group key of the previous session; similarly, the leaving protocol cannot provide group forward secrecy so that leaving members can get the next session key. We present the security flaws of these protocols and propose our new joining/leaving protocols to guarantee group forward/backward secrecy.

Our paper is organized as follows: The preliminaries are given in section II, and the review on Wan et al.'s protocols is described in section III. In section IV, we show the security weaknesses of Wan et al.'s protocols. Then we present our joining/leaving protocols in section $\mathrm{V}$ and analyze our scheme in section VI. We finally conclude our paper in section VII.

\section{Preliminaries}

\subsection{Security Requirements}

Because GKA protocols can suffer from passive or active adversaries, we have to consider the requirements for designing GKA protocols to protect the identities or the communication of group members from those adversaries. In case of anonymous ID-based GKA protocol, it becomes more complicated that anonymity and unlinkability should be provided. Wan et al. already defined the requirements for their anonymous ID-based GKA protocol; additionally, we consider one more requirement, entity authentication, because each legitimate group member should have confidence that the other members are really participating in the protocol while the protocol provides anonymity. The security requirements for the GKA protocols are as follows:

- Anonymity: The communication messages do not carry 
any information about group members' identities for protecting the identities from the outside eavesdropper.

- Unlinkability : The group members' activities in two different sessions must be independent; in other words, all the sessions are unlinkable to each other.

- Group Key Secrecy : Any adversary cannot compute the session group key.

- Group Forward Secrecy : Any adversary (especially the leaving member) who knows the previous group key cannot obtain the subsequent group key and communication messages.

- Group Backward Secrecy : Any adversary (especially the joining member) who knows the current group key cannot obtain the preceding group key and communication messages.

- Perfect Forward Secrecy : Revealing the long-term secret key does not affect the secrecy of the established session keys from previous protocol sessions.

- Entity Authentication : Each group member should have confidence that the other members are actually involved in the protocol.

\subsection{Bilinear Pairing}

Let $G_{1}$ and $G_{2}$ be cyclic additive and multiplicative groups of prime order $q$, respectively. A mapping $e$ : $G_{1} \times G_{1} \rightarrow G_{2}$ which satisfies the following properties is called bilinear pairing:

1) Bilinearity : $e(a P, b Q)=e(P, Q)^{a b}$ for all $P, Q \in G_{1}$ and $a, b \in Z_{q}^{*}$.

2) Non-degeneracy : If a generator $P$ belongs to $G_{1}$, then $e(P, P)$ is a generator of $G_{2}$; in other words, $e(P, P) \neq$ 1.

3) Computable : An efficient algorithm to compute $e(P, Q)$ exists for any $P, Q \in G_{1}$.

\subsection{Adversarial Model}

There are two types of adversaries: passive and active adversaries. The ability of a passive adversary is restricted to eavesdropping communications only, but an active adversary additionally can replace, modify, or intercept messages. The goals of adversaries in GKA protocols are computing the subset of group keys or impersonation of the legitimate group member.

To provide computational security, we introduce two computationally infeasible problems, Bilinear Diffie-Hellman (BDH) and Elliptic Curve Diffie-Hellman (ECDH) problems.

- BDH Problem: Given $P, a P, b P$, and $c P$, compute $e(P, P)^{a b c}$ where $P \in G_{1}, a, b, c \in Z_{q}^{*}$, and $e$ is a bilinear pairing.

- ECDH Problem: Given $P, a P$, and $b P$, compute $a b P$ where $P$ is an element of an elliptic curve and $a, b \in Z_{q}^{*}$ $\mathrm{BDH} / \mathrm{ECDH}$ assumptions state that $\mathrm{BDH} / \mathrm{ECDH}$ problems are hard to solve.

\section{Review on Wan et al.'s Anonymous GKA Protocol}

In this section, we review Wan et al.'s anonymous IDbased GKA protocol and joining/leaving protocols for single user operation in a specific group.

\subsection{Setup}

Wan et al.'s protocol is based on Boneh and Franklin's ID-based cryptosystem setup [2] using bilinear pairing. To start setup phase, a trusted $\mathrm{KGC}$ chooses a random $s \in Z_{q}^{*}$ as the secret master key, and generates the system parameters: param $=<G_{1}, G_{2}, q, e, P, P_{\text {pub }}, H_{1}>$,

where $P$ is an arbitrary generator of $G_{1}$, and $H_{1}$ is a hash function, $H_{1}:\{0,1\}^{*} \rightarrow Z_{q}^{*}$.

Then KGC produces the public key $Q_{I D}=H_{1}(I D)$ and the private key $S_{I D}=s Q_{I D}$ using the a user's identity $I D$. For instance, a user with identity $U_{i}$ has the static key pair $<Q_{i}, S_{i}>$.

The notations used in Wan et al.'s protocol are as follows:

\begin{tabular}{c|l}
$E_{i}(*)$ & ID-based encryption using $U_{i}$ \\
$E_{K}(*)$ & Symmetric encryption using $K$ \\
$N y m_{i}$ & Pseudonym for $U_{i}$ \\
$r_{i}$ & Random number selected by $U_{i}$ \\
$S I G_{i}$ & $U_{i}$ 's signature \\
$h$ & A hash function $h: G_{2} \times G_{1} \rightarrow\{0,1\}^{k}$ \\
& with a security parameter $k$
\end{tabular}

\subsection{GKA Protocol}

There are $n$ entities in Wan et al.'s GKA protocol: a group initiator $U_{1}$ and the other group members $U_{2}, \ldots, U_{n}$. The initiator $U_{1}$, who knows all the identities of the other members, initiates a new session for starting the GKA protocol. The other members do not know the identities of the group members before the session starts. The protocol uses the public system parameter set param.

1) Initiator $U_{1}$ chooses pseudonyms for each user $U_{i}$. $U_{1} \rightarrow U_{i}: E_{i}\left(U_{1}\left\|\ldots|| U_{n}\right\| N \operatorname{Nm}_{1}\|\ldots\| N y m_{n} \| S I G_{1}\right)$, $r_{1} P$

2) $U_{i(\neq 1)}$ sends a message to $U_{i-1}$ and $U_{i+1}$.

$U_{i} \rightarrow U_{i+1}, U_{i-1}:$ Nym $_{i}, r_{i} P$

3) $U_{i}$ verifies the pseudonyms, and computes

$$
\begin{aligned}
& k_{i}=h\left(e\left(Q_{i+1}, S_{i}\right) \| r_{i} r_{i+1} P\right) \\
& k_{i-1}=h\left(e\left(Q_{i-1}, S_{i}\right) \| r_{i} r_{i-1} P\right) .
\end{aligned}
$$$$
U_{i} \rightarrow *: \text { Nym }_{i}, X_{i}=k_{i} / k_{i-1}
$$

4) $U_{i}$ verifies all the pseudonyms, and computes

$$
\begin{aligned}
k_{i+1}=k_{i} X_{i+1}, k_{i+2} & =k_{i+1} X_{i+2}, \\
\ldots, k_{i+n-1} & =k_{i+n-1} X_{i+n-1} .
\end{aligned}
$$

Session Key : $K=H\left(k_{1}|| k_{2} \| . .|| k_{n}\right)$

After computing the session group key $K, U_{i(\neq 1)}$ sends $H\left(K\left\|U_{1}|| U_{2}\right\| \ldots \| U_{n}\right)$ to $U_{1}$. Then $U_{1}$ verifies whether all the other group members computed the same key or not. 


\subsection{Joining Protocol}

In the joining protocol, $U_{1}$ firstly informs $U_{n+1}$ 's joining. Then only $U_{1}$ and $U_{n}$, who become $U_{n+1}$ 's neighbors, compute $X_{1}^{\prime}$ and $X_{n}^{\prime}$ to generate a new session group key. The protocol description is as follows:

1) $U_{1}$ informs $U_{n}$ and $U_{n+1}$ about joining information.

$$
\begin{aligned}
U_{1} & \rightarrow U_{n}: E_{n}\left(U_{n+1}\left\|N y m_{n+1}\right\| S I G_{1}\right) \\
U_{1} & \rightarrow U_{n+1}: E_{n+1}\left(U_{1}\left\|N m_{1}\right\| r_{1} P \| U_{n}\right. \\
& \left.\quad\left\|N m_{n}\right\| r_{n} P\left\|U_{n+1}\right\| N y m_{n+1} \| S I G_{1}\right)
\end{aligned}
$$

2) $U_{n+1}$ computes

$$
\begin{gathered}
k_{n+1}=h\left(e\left(Q_{1}, S_{n+1}\right) \| r_{1} r_{n+1} P\right) \\
k_{n}^{\prime}=h\left(e\left(Q_{n}, S_{n+1}\right) \| r_{n} r_{n+1} P\right) . \\
X_{n+1}=k_{n+1} / k_{n}^{\prime} \\
U_{n+1} \rightarrow U_{1}, U_{n}: \text { Nym }_{n+1}, r_{n+1} P, X_{n+1}
\end{gathered}
$$

3) $U_{1}$ and $U_{n}$ compute

$$
\begin{gathered}
U_{1}: k_{n+1}=h\left(e\left(Q_{n+1}, S_{1}\right) \| r_{1} r_{n+1} P\right), \\
X_{1}^{\prime}=k_{1} / k_{n+1} \\
U_{n}: k_{n}^{\prime}=h\left(e\left(Q_{n+1}, S_{n}\right) \| r_{n} r_{n+1} P\right), \\
X_{n}^{\prime}=k_{n} / k_{n-1} . \\
U_{n} \rightarrow U_{1}: X_{n}^{\prime}
\end{gathered}
$$

4) $U_{1}$ informs all the members about changed information.

$$
\begin{aligned}
& U_{1} \rightarrow U_{n+1}: E_{n+1}\left(X_{1}^{\prime}\left\|X_{2}\right\| \ldots\left\|X_{n-1}\right\| X_{n}^{\prime}\right) \\
& U_{1} \rightarrow *: E_{K}\left(X_{1}^{\prime}\left\|X_{n+1}\right\| X_{n}^{\prime} \| S I G_{1}\right) \\
& \text { Session Key : } K^{\prime}=H\left(k_{1}\left\|k_{2}\right\| . .\left\|k_{n}^{\prime}\right\| k_{n+1}\right)
\end{aligned}
$$

The group members, except $U_{1}$ and $U_{n}$, do not need to compute $X_{i}$ again during the joining protocol.

\subsection{Leaving Protocol}

In the leaving protocol, $U_{1}$ informs $U_{l}$ 's leaving. Then $U_{l-1}$ and $U_{l+1}$, who were $U_{l}$ 's neighbors in the previous session, compute $X_{l-1}^{\prime}$ and $X_{l+1}^{\prime}$ to generate a new session group key without $U_{l}$. The protocol description is as follows:

1) $U_{1}$ informs $U_{l-1}$ and $U_{l+1}$ about leaving information.

$$
\begin{array}{r}
U_{1} \rightarrow U_{l-1}, U_{l+1}: E_{K}\left(U_{l}\left\|N y m_{l}\right\| U_{l-1} \|\right. \\
\left.\quad N y m_{l-1}^{\prime}\left\|U_{l+1}\right\| N y m_{l+1}^{\prime} \| S I G_{1}\right)
\end{array}
$$

2) $U_{l-1}$ and $U_{l+1}$ exchange their new random values.

$U_{l-1} \rightarrow U_{l+1}: N y m_{l-1}^{\prime}, r_{l-1} P$
$U_{l+1} \rightarrow U_{l-1}: N y m_{l+1}^{\prime}, r_{l+1} P$

3) $U_{l-1}$ and $U_{l+1}$ compute

$$
\begin{aligned}
U_{l-1}: & k_{l-1}^{\prime}=h\left(e\left(Q_{l+1}, S_{l-1}\right) \| r_{l-1}^{\prime} r_{l+1}^{\prime} P\right), \\
& X_{l-1}^{\prime}=k_{l-1}^{\prime} / k_{l-2} \\
U_{l+1}: & k_{l}^{\prime}=h\left(e\left(Q_{l-1}, S_{l+1}\right) \| r_{l-1}^{\prime} r_{l+1}^{\prime} P\right), \\
& X_{l+1}^{\prime}=k_{l+1} / k_{l-1}^{\prime} . \\
U_{l-1} \rightarrow & U_{1}: X_{l-1}^{\prime} \\
U_{l+1} \rightarrow & U_{1}: X_{l+1}^{\prime}
\end{aligned}
$$

4) $U_{1}$ informs all the members about changed information. $U_{1} \rightarrow *: E_{K}\left(U_{l}\left\|U_{l-1}\right\| U_{l+1}\left\|X_{l-1}^{\prime}\right\| X_{l+1}^{\prime} \| S I G_{1}\right)$ Session Key : $K^{\prime}=H\left(k_{1}\|\ldots\| k_{l-1}^{\prime}|| k_{l+1}\|\ldots\| k_{n}\right)$

\section{Cryptanalysis on Wan et al.'s Protocols}

Wan et al.'s GKA protocol is insecure in the presence of malicious group participants. Moreover, their joining and leaving protocols also have security weaknesses. In this section, we show these weaknesses of the protocols.

\subsection{Impersonation by Colluding Attack in GKA Protocol}

To show an attack on Wan et al.'s GKA protocol, we assume that the malicious users $U_{m-1}$ and $U_{m+1}$ can collude with the group initiator $U_{1}$ and want to impersonate the group member $U_{m}$. When starting the GKA protocol, $U_{1}$ sends group information to the other group members except $U_{m}$, and sends one additional random value to $U_{m-1}$ and $U_{m+1}$, who are two neighbors of $U_{m}$. Using this information, $U_{m-1}$ and $U_{m+1}$ can easily impersonate $U_{m}$ without any private information of $U_{m}$. A detailed description of the attack is as follows:

1) $U_{1}$ chooses pseudonyms for each user $U_{i}$.

$$
\begin{gathered}
U_{1} \rightarrow U_{i(\neq m)}: E_{i}\left(U_{1}\|\ldots\| U_{n} \|\right. \\
\left.\quad N_{1}\|\ldots\| N m_{1}\left\|\ldots m_{n}\right\| S I G_{1}\right), r_{1} P \\
U_{1} \rightarrow U_{m-1}, U_{m+1}: r_{m} P
\end{gathered}
$$

2) $U_{m-1}$ and $U_{m+1}$ get pseudonyms and send the random value only to $U_{m-2}$ and $U_{m+2}$, while the other members send their random values to their two neighbors.

$$
\begin{aligned}
& U_{i} \rightarrow U_{i-1}, U_{i+1}: N y m_{i}, r_{i} P \\
& U_{m+1} \rightarrow U_{m+2},\left(\text { not } U_{m}\right): N y m_{m+1}, r_{m+1} P \\
& U_{m-1} \rightarrow U_{m-2},\left(\text { not } U_{m}\right): N y m_{m-1}, r_{m-1} P
\end{aligned}
$$

3) $U_{m-1}$ and $U_{m+1}$ can compute $k_{m}$ and $k_{m-1}$ which are originally generated by $U_{m}$.

$$
\begin{aligned}
& U_{m+1}: k_{m}=h\left(e\left(Q_{m}, S_{m+1}\right) \| r_{m} r_{m+1} P\right) \\
& U_{m-1}: k_{m-1}=h\left(e\left(Q_{m}, S_{m-1}\right) \| r_{m} r_{m-1} P\right) .
\end{aligned}
$$$$
U_{m+1} \text { or } U_{m-1} \rightarrow *: \text { Nym }_{m}, X_{m}=k_{m} / k_{m-1}
$$

4) If each $U_{i}$ succeeds in verifying all the pseudonyms, then computes

$$
\begin{aligned}
& k_{i+1}=k_{i} X_{i+1}, k_{i+2}=k_{i+1} X_{i+2} \text {, } \\
& \ldots, k_{i+n-1}=k_{i+n-1} X_{i+n-1} \text {. } \\
& \text { Session Key : } K=H\left(k_{1}|| k_{2} \| . .|| k_{n}\right)
\end{aligned}
$$

Through this attack, the other group members cannot recognize $U_{m}$ 's missing and just generate a session group key without $U_{m}$. This attack is possible because the security of messages depends on that of pseudonyms, and group members do not authenticate whether the message is actually generated by the specific member or not. Note that the computation of $X_{m}$ can be computed not only by the $U_{m}$ but also by $U_{m-1}$ and $U_{m+1}$. Therefore, malicious users $U_{m-1}$ and $U_{m+1}$ can impersonate the user $U_{m}$. To prevent this attack, each member should contain a signature while broadcasting $X_{i}$. If the members verify all the other members' signatures, they easily know $U_{m}$ 's missing and stop the protocol. We recommend using Cheon et al.'s IDbased signature [4], which provides batch verification. With this scheme, users can reduce the authentication cost by verifying several signatures at once. 


\subsection{Weakness on Backward Secrecy in the Joining Protocol}

We also prove that Wan et al.'s joining protocol cannot provide backward secrecy. In their joining protocol, we assume that joining member $U_{n+1}$ can obtain previous transcripts; then $U_{n+1}$ can compute not only a new group key $K^{\prime}$ but also the previous group key $K$, which is used before $U_{n+1}$ joins the group.

During the joining protocol execution, $U_{n+1}$ computes a new session key $K^{\prime}$. Equations for key generation in the GKA and joining protocols are as follows:

Previous key: $K=H\left(k_{1}\left\|k_{2}\right\| . . \mid k_{n}\right)$

New key: $K^{\prime}=H\left(k_{1}\left\|k_{2}\right\| . .|| k_{n-1}|| k_{n}^{\prime} \| k_{n+1}\right)$

In the new session group key, only $k_{n}$ is changed from the previous session key, so $U_{n+1}$ has all information about $K$, except $k_{n}$. If $U_{n+1}$ can obtain $k_{n}$, then he also can compute the previous group key $K$. Here, $U_{n+1}$ can extract $k_{n}=k_{n-1} X_{n}$ using the previous transcript $<N y m_{n}, X_{n}>$ because it was broadcasted in the previous session. Therefore, $U_{n+1}$ can compute the previous group key, $K=H\left(k_{1}\left\|k_{2}\right\| . . \| k_{n}\right)$.

Through this procedure, a joining member can compute the previous group key, using the previous transcript and the current session group key. That is, we can prove that the joining protocol cannot guarantee backward secrecy.

\subsection{Weakness on Forward Secrecy in the Leaving Protocol}

Here we show that Wan et al.'s leaving protocol cannot provide forward secrecy. When $U_{l}$ leaves the group, the other members generate a new session group key $K^{\prime}$ with changed information. Equations for key generation in the GKA and leaving protocols are as follows:

Previous key: $K=H\left(k_{1}\left\|k_{2}\right\| . .|| k_{n}\right)$

New key: $K^{\prime}=H\left(k_{1}\left\|\ldots|| k_{l-1}^{\prime}\right\| k_{l+1}\|\ldots\| k_{n}\right)$

Because only $k_{l-1}$ is changed to $k_{l-1}^{\prime}$ in the new session group key, $U_{l}$ has all information about $K^{\prime}$, except $k_{l-1}^{\prime}$. If $U_{l}$ can obtain $k_{l-1}^{\prime}$, then he also can compute the new session group key $K^{\prime}$. In the protocol, however, $U_{1}$ informs all the members about changed information as follows:

$$
U_{1} \rightarrow *: E_{K}\left(U_{l}\left\|U_{l-1}\right\| U_{l+1}\left\|X_{l-1}^{\prime}\right\| X_{l+1}^{\prime} \| S I G_{1}\right)
$$

The message is encrypted using the previous group key $K$, so $U_{l}$ can decrypt this message to get $k_{l-1}^{\prime}=k_{l-2} X_{l-1}^{\prime}$; consequently, he can generate the new session key $K^{\prime}$.

Through this procedure, a leaving member still can compute a new session group key although he no longer belongs to the group. Therefore, we can prove that the leaving protocol cannot guarantee forward secrecy.

\section{Our Proposed Scheme}

Here we propose our new joining/leaving protocol of Wan et al.'s GKA protocol.

\subsection{Joining Protocol}

In Wan et al.'s joining protocol, all the $k_{i}$ 's except $k_{n}$ are reused to generate a new session group key; therefore, a joining member who obtain the previous transcript can compute the previous group key. To deal with this problem, all the $k_{i}$ 's should be changed for each session, and the new group key should not contain information of the previous session. Computation of $k_{i}$, however, requires pairing computation which takes comparably high cost. Considering this fact, we design our joining protocol reducing the cost of computing $k_{i}^{\prime}$. We define two hash functions $g:\{0,1\}^{k} \rightarrow Z_{q}^{*}$, where $|q|=2^{k}-1$, and $H_{2}: G_{1} \rightarrow\{0,1\}^{k}$.

1) Initiator $U_{1}$ informs all the group members about $U_{n+1}$ 's joining.

$$
\begin{aligned}
U_{1} \rightarrow *: E_{K} & \left(U_{n+1}\left\|N y m_{1}^{\prime}\right\| \ldots \|\right. \\
& \left.N y m_{n}^{\prime}\left\|N y m_{n+1}\right\| S I G_{1}\right) \\
U_{1} \rightarrow U_{n+1}: & E_{n+1}\left(U_{1}\|\ldots\| U_{n}\left\|U_{n+1}\right\| N y m_{1}^{\prime}\|\ldots\|\right. \\
& \left.N y m_{n}^{\prime}\left\|N y m_{n+1}\right\| r_{1} P\left\|r_{n} P\right\| S I G_{1}\right)
\end{aligned}
$$

2) $U_{n+1}$ computes

$$
\begin{aligned}
& k_{n+1}=h\left(e\left(Q_{1}, S_{n+1}\right) \| r_{1} r_{n+1} P\right) \\
& k_{n}^{\prime}=h\left(e\left(Q_{n}, S_{n+1}\right) \| r_{n} r_{n+1} P\right) \\
& X_{n+1}=k_{n+1} / k_{n}^{\prime} .
\end{aligned}
$$$$
U_{n+1} \rightarrow U_{1}, U_{n}: N y m_{n+1}, r_{n+1} P, X_{n+1}
$$

3) $U_{i}$ computes

$$
\begin{aligned}
& k_{i}^{\prime}=H_{2}\left(g\left(k_{i}\right) r_{i} r_{i+1} P\right), k_{i-1}^{\prime}=H_{2}\left(g\left(k_{i}\right) r_{i} r_{i-1} P\right), \\
& \text { and } U_{1} \text { and } U_{n} \text { compute } \\
& U_{1}: k_{n+1}=h\left(e\left(Q_{n+1}, S_{1}\right) \| r_{1} r_{n+1} P\right), \\
& \quad k_{1}^{\prime}=H_{2}\left(g\left(k_{1}\right) r_{1} r_{2} P\right) \\
& U_{n}: k_{n}^{\prime}=h\left(e\left(Q_{n+1}, S_{n}\right) \| r_{n} r_{n+1} P\right), \\
& \quad k_{n-1}^{\prime}=H_{2}\left(g\left(k_{n}\right) r_{n} r_{n-1} P\right) . \\
& U_{i} \rightarrow *: N y m_{i}^{\prime}, X_{i}^{\prime}=k_{i}^{\prime} / k_{i-1}^{\prime}, S I G_{i} \\
& \text { Session Key: } K^{\prime}=H\left(k_{1}^{\prime}\left\|k_{2}^{\prime}\right\| . .\left\|k_{n}^{\prime}\right\| k_{n+1}\right)
\end{aligned}
$$

The $k_{i}$ 's are changed in each session, so $U_{n+1}$ cannot extract the previous session group key even if he has the previous transcripts. Additionally, when broadcasting $X_{i}^{\prime}$ to other users, all users contain their signatures as recommended in section 4.1 .

\subsection{Leaving Protocol}

As in the joining protocol, all the $k_{i}$ 's except $k_{l-1}$ are used to generate a new session group key in Wan et al.'s leaving protocol. Moreover, significant information to generate the new session group key is encrypted using the previous group key $K$, so leaving members can compute the new session group key $K^{\prime}$. Two ways to solve this weakness are recomputing all the $k_{i}$ 's and not using symmetric encryption with previous group key $K$ for significant information. The protocol procedure is as follows:

1) Initiator $U_{1}$ informs all the other members about $U_{l}$ 's leaving.

$U_{1} \rightarrow *: E_{K}\left(U_{l}\left\|N y m_{1}^{\prime}\right\| \ldots\left\|N y m_{n}^{\prime}\right\| S I G_{1}\right)$

2) $U_{l-1}$ and $U_{l+1}$ exchange their new random values. 


$$
\begin{gathered}
U_{l-1} \rightarrow U_{l+1}: N y m_{l-1}^{\prime}, r_{l-1} P \\
U_{l+1} \rightarrow U_{l-1}: N y m_{l+1}^{\prime}, r_{l+1} P \\
\text { 3) } U_{i} \text { computes } \\
k_{i}^{\prime}=H_{2}\left(g\left(k_{i}\right) r_{i} r_{i+1} P\right), k_{i-1}^{\prime}=H_{2}\left(g\left(k_{i}\right) r_{i} r_{i-1} P\right), \\
\text { and } U_{i-1} \text { and } U_{i+1} \text { compute } \\
U_{l-1}: k_{l-1}^{\prime}=h\left(e\left(Q_{l+1}, S_{l-1}\right) \| r_{l-1}^{\prime} r_{l+1}^{\prime} P\right), \\
k_{l-2}^{\prime}=H_{2}\left(g\left(k_{l-1}\right) r_{l-1} r_{l-2} P\right) \\
U_{l+1}: k_{l-1}^{\prime}=h\left(e\left(Q_{l-1}, S_{l+1}\right) \| r_{l-1}^{\prime} r_{l+1}^{\prime} P\right), \\
k_{l+1}^{\prime}=H_{2}\left(g\left(k_{l+1}\right) r_{l+1} r_{l+2} P\right) . \\
U_{i} \rightarrow *: N y m_{i}^{\prime}, X_{i}^{\prime}=k_{i}^{\prime} / k_{i-1}^{\prime}, S I G_{i} \\
\text { Session Key: } K^{\prime}=H\left(k_{1}^{\prime}\left\|\ldots k_{i-1}^{\prime}\right\| k_{i+1}^{\prime}\|\ldots\| k_{n}^{\prime}\right)
\end{gathered}
$$

The $k_{i}$ 's are changed in each session, so $U_{l}$ cannot compute the later session group key even if he has all the previous $k_{i}$ 's. Also, the signature is included when each group member broadcasts $X_{i}^{\prime}$ to other users.

\section{Analysis}

In the previous sections, we recommended including signature during Wan et al.'s GKA protocol and proposed our new joining/leaving protocols. Here, we analyze security and performance of our scheme in detail.

\subsection{Security}

As already explained, there are two types of adversaries: passive and active adversaries. From eavesdropping the protocol execution, the passive adversary can get $N y m_{i}$, $r_{i} P$, and $X_{i}$ values. With this information, the adversary should not be able to get any information about the group members or the session group key. The active adversary want to interrupt the protocol execution or to impersonate the legitimate group member with more information than the passive adversary. This type of adversary additionally can obtain $k_{i}$ 's or the public/private key pair $<Q_{i}, S_{i}>$ of the group member. In the case that the adversary gets the public/private key pair of the group member, he should not be able to compute the previous group keys. Also, the joining/leaving group member who gets the current or previous $k_{i}$ 's should not be able to compute the precedeing/subsequent group keys.

a) Anonymity : In the GKA protocol, the message firstly sent from the initiator is encrypted using the private key of each member, and only the legitimate group members can decrypt this message and get the pseudonyms. Even though an outside eavesdropper obtains all the pseudonyms from the transcript, the eavesdropper cannot match them to the real identities of members unless he can decrypt the message using the private key.

Similarly, the informing messages sent from the initiator are encrypted using the group key of the previous session in the joining/leaving protocols.

- Join : $E_{K}\left(U_{n+1}\left\|N y m_{1}^{\prime}\right\| \ldots|| N y m_{n}^{\prime}\left\|N y m_{n+1}\right\| S I G_{1}\right)$

- Leave : $E_{K}\left(U_{l}\left\|N y m_{1}^{\prime}\right\| \ldots\left\|N y m_{n}^{\prime}\right\| S I G_{1}\right)$
The eavesdropper cannot get the pseudonyms of all the group members without the previous session group key $K$. Therefore, the protocols keep the group members anonymous to outside eavesdroppers.

b) Unlinkability : When the session starts, the initiator always generates pseudonyms for the group members (also in the joining/leaving protocols); that is, the pseudonyms are never reused. Although the adversary wants to trace user information using all the pseudonyms of different sessions, he cannot link them to any user information because pseudonyms always change in each session and do not carry any information about the group members' identities.

c) Group Key Secrecy : In the GKA protocol, the group key $K$ is generated by concatenating all the $k_{i}$ 's. Because the $k_{i}$ 's are obtained sequentially with one $k_{i}$ and all the other $X_{i}$ 's, the adversary should have at least one $k_{i}$ to compute the session group key. However, when computing $k_{i}$, it is difficult to compute $r_{i} r_{i+1} P$ given $<P, r_{i} P, r_{i+1} P>$ tuple under the ECDH assumption; also computing $e\left(Q_{i+1}, S_{i}\right)$ without the master secret key $s$ is a hard problem under the $\mathrm{BDH}$ assumption. Therefore, the passive adversary cannot compute the group key $K$.

d) Group Forward Secrecy : Our leaving protocol provides group forward secrecy when a user leaves the group; in other words, the $U_{l}$ cannot compute the subsequent group key. In our protocol, all the $k_{i}$ 's changes in each session and no symmetric encryption is used to encrypt new $X_{i}$ 's, so $U_{l}$ cannot extract $k_{i}^{\prime}$ using $k_{i}$. Also, under the ECDH assumption, it is hard to compute $r_{i} r_{i+1} P$ given $<P, r_{i} P$, $r_{i+1} P>$ tuple when computing $k_{i}^{\prime}=H_{2}\left(g\left(k_{i}\right) r_{i} r_{i+1} P\right)$; therefore, $U_{l}$ cannot compute $k_{i}^{\prime}$ although he has all the previous $k_{i}$ and $r_{i} P$. Through this result, we can prove that our leaving protocol provides group forward secrecy.

e) Group Backward Secrecy : Our joining protocol provides group backward secrecy when a user joins the group; that is, the $U_{n+1}$ cannot compute the preceding group key. In our protocol, all the $k_{i}$ 's changes for each session, so $U_{n+1}$ cannot extract these values using the previous transcript. A joining member must compute $k_{i}$ again with gathered information to compute the previous group key, but it is impossible to extract $k_{i}$ from $k_{i}^{\prime}=H_{2}\left(g\left(k_{i}\right) r_{i} r_{i+1} P\right)$. Therefore, joining members cannot compute previous group keys; in other words, our joining protocol provides group backward secrecy.

f) Perfect Forward Secrecy: In our protocol, the computation of $k_{i}$ needs public/private key pair and $r_{i} r_{i+1} P$. Although the adversary reveals the private key $S_{i}$, he cannot compute $k_{i}$ because computing $r_{i} r_{i+1} P$ given $\left\langle P, r_{i} P, r_{i+1} P>\right.$ is hard problem under the ECDH assumption. Therefore, our 
Table 1. Comparison: Joining Protocols

\begin{tabular}{|c|c|c|c|c|c|c|c|c|c|}
\hline & & $I D$ & Sig & Sym & $P$ & $M$ & $D$ & $B$ & $U$ \\
\hline \multirow{3}{*}{ [7] } & $U_{1}$ & 3 & 3 & 1 & 1 & 1 & 1 & 1 & 3 \\
\hline & $U_{n}$ & 0 & 0 & 0 & 1 & 1 & 1 & 0 & 1 \\
\hline & $U_{n+1}$ & 0 & 0 & 0 & 2 & 2 & 1 & 0 & 2 \\
\hline \multirow{4}{*}{ Ours } & $\overline{U_{1}}$ & 1 & 2 & 1 & 1 & 2 & 1 & 2 & 1 \\
\hline & $U_{n}$ & 0 & 1 & 0 & 1 & 2 & 1 & 1 & 0 \\
\hline & $U_{n+1}$ & 0 & 1 & 0 & 2 & 2 & 1 & 0 & 2 \\
\hline & $U_{i}$ & 0 & 1 & 0 & 0 & 2 & 1 & 1 & 0 \\
\hline
\end{tabular}

protocol provides perfect forward secrecy that revealing long-term keying material does not affect the secrecy of the established keys from previous sessions.

g) Entity Authentication: When the group members broadcast $X_{i}$ 's in Wan et al.'s protocols, they verify that value with only the pseudonym $N y m_{i}$. This verification causes user impersonation of the malicious participants who know the pseudonyms. If the group members generate ID-based signatures for $X_{i}$ and the other members verify all the signatures, they can easily authenticate the other users. Therefore, our protocol can provide entity authentication with the verification of the ID-based signatures that we recommended in section 4.1. (In this case, the security by entity authentication depends on the security of the ID-based signature.)

\subsection{Performance}

Tables 1 and 2 show the comparison of Wan et al.'s protocols [7] and our proposed protocols in performance. We use the following notations:

ID: Number of ID-based encryption using $Q_{i} / S_{i}$ Sig: Number of ID-based signature using $Q_{i} / S_{i}$ Sym: Number of Symmetric encryption using $K$ $P$ : Number of pairing computation for each user $M$ : Number of multiplication for each user

$D$ : Number of division for each user

$B$ : Number of broadcast for each user

$U$ : Number of unicast for each user

Although all users should compute their $k_{i}$ for each session in our joining/leaving protocols, the computation of new $k_{i}$ requires only 2 scalar multiplications, 1 division, and 1 broadcast. Moreover, in Wan et al.'s joining protocol, $U_{1}$ should compute 4 encryptions for generating new session group key, but only 2 encryptions are required in our joining protocol. Therefore, our joining/leaving protocols does not increase much computation cost from Wan et al.'s protocols.

\section{Conclusion}

In this paper, we found security weaknesses in Wan et al.'s ID-based GKA protocol and joining/leaving protocols:
Table 2. Comparison: Leaving Protocols

\begin{tabular}{|c|c|c|c|c|c|c|c||c|c|}
\hline & & ID & Sig & Sym & $P$ & $M$ & $D$ & $B$ & $U$ \\
\hline \hline$[7]$ & $U_{1}$ & 0 & 2 & 2 & 0 & 0 & 0 & 0 & 2 \\
& $U_{l \pm 1}$ & 0 & 0 & 0 & 1 & 2 & 1 & 0 & 2 \\
\hline \hline \multirow{3}{*}{ Ours } & $U_{1}$ & 0 & 1 & 1 & 0 & 2 & 1 & 2 & 0 \\
& $U_{l \pm 1}$ & 0 & 1 & 0 & 1 & 3 & 1 & 1 & 1 \\
$U_{i}$ & 0 & 1 & 0 & 0 & 2 & 1 & 1 & 0 \\
\hline
\end{tabular}

the GKA protocol suffers from insider colluding attack, and joining/leaving protocols cannot guarantee group backward/forward secrecy. We recommended using the ID-based signature to prevent the attack on the GKA protocol and proposed our joining/leaving protocols. In our protocols, all the group members must recompute individual secret, $k_{i}$, for each session to generate a new session group key, so no joining or leaving member can obtain the previous or later session group key with the previous individual secrets. In other words, our protocols can provide group forward/backward secrecy. Additionally, our joining/leaving protocols can operate efficiently compared with the previous protocol. With the our joining/leaving protocols and the GKA protocol containing ID-based signature, the security can be enhanced while comparable efficiency is maintained.

\section{References}

[1] A. Shamir, Identity-based Cryptosystems and Signature Schemes, Advances in Cryptology-Crypto 84, LNCS 196, pp.47-53, Springer-Verlag, 1984.

[2] D. Boneh and M. Franklin, Identity-based encryption from the Weil pairing, Proc. of Crypto'01, LNCS 2139, pp.213-229, Springer-Verlag, 2001.

[3] K. Y. Choi, J. Y. Hwang and D. H. Lee, Efficient ID-based Group Key Agreement with Bilinear Maps. PKC'04, LNCS 2947, pp.130-144, Springer-Verlag, 2004.

[4] J. H. Cheon, and Y. Kim, H. Yoon. A New ID-based Signature with Batch Verification, Cryptology ePrint Archive, Report 2004/131.

[5] Y. Shi, G. Chen, J. Li. ID-Based One Round Authenticated Group Key Agreement Protocol with Bilinear Pairings, International Conference on Information Technology: Coding and Computing (ITCC'05),--Volume I pp.757-761, 2005.

[6] L. Zhou, W. Susilo, Y. Mu. Efficient ID-based Authenticated Group Key Agreement from Bilinear Pairings, Mobile Ad-hoc and Sensor Networks -MSN 2006, LNCS 4325, pp. 521-532, Springer-Verlag, 2006.

[7] Z. Wan, K. Ren, W. Lou, B. Preneel, Anonymous IDbased Group Key Agreement for Wireless Networks, Wireless Communications and Networking Conference-2008 (WCNC 2008),IEEE , pp.2615-2620, 2008.

[8] G. Yao, H. Wang, Q. Jiang. An Authenticated 3-Round IdentityBased Group Key Agreement Protocol, In proc. of the third International Conference on Availability, Reliability, and Security -ARES'08, pp. 538-543, ACM, 2008. 